\title{
Targeting plasma membrane phosphatidylserine content to inhibit oncogenic KRAS function
}

\author{
Walaa E Kattan ${ }^{1,3}$, Wei Chen ${ }^{1}$, Xiaoping Ma ${ }^{1}$, Tien Hung Lan ${ }^{1}$, Dharini van der Hoeven², Ransome van der Hoeven², \\ John F Hancock ${ }^{1,3}$ (D)
}

\begin{abstract}
The small GTPase KRAS, which is frequently mutated in human cancers, must be localized to the plasma membrane (PM) for biological activity. We recently showed that the KRAS C-terminal membrane anchor exhibits exquisite lipid-binding specificity for select species of phosphatidylserine (PtdSer). We, therefore, investigated whether reducing PM PtdSer content is sufficient to abrogate KRAS oncogenesis. Oxysterol-related binding proteins ORP5 and ORP8 exchange PtdSer synthesized in the ER for phosphatidyl-4-phosphate synthesized in the PM. We show that depletion of ORP5 or ORP8 reduced PM PtdSer levels, resulting in extensive mislocalization of KRAS from the PM. Concordantly, ORP5 or ORP8 depletion significantly reduced proliferation and anchorage-independent growth of multiple KRAS-dependent cancer cell lines, and attenuated KRAS signaling in vivo. Similarly, functionally inhibiting ORP5 and ORP8 by inhibiting PI4KIII $\alpha$ mediated synthesis of phosphatidyl-4-phosphate at the PM selectively inhibited the growth of KRAS-dependent cancer cell lines over normal cells. Inhibiting KRAS function through regulating PM lipid PtdSer content may represent a viable strategy for KRAS-driven cancers.
\end{abstract}

DOI 10.26508/lsa.201900431 | Received 17 May 2019 | Revised 16 August 2019 | Accepted 19 August 2019 | Published online 26 August 2019

\section{Introduction}

RAS proteins are membrane-localized GTPases that regulate cell proliferation, differentiation, and apoptosis. RAS is a molecular switch that oscillates between an active GTP-bound and inactive GDP-bound state and functions as a critical node in growth factor receptor signaling pathways. Two classes of proteins regulate RAS.GTP levels: guanine nucleotide exchange factors activate RAS by promoting exchange of GDP for GTP, and GTPase-activating proteins stimulate RAS GTPase activity to return RAS.GTP to the inactive ground state. This regulatory circuit is subverted in 15-20\% of all human tumors that express oncogenic RAS with mutations at residues 12, 13, or 61 (Cox et al, 2014). These mutations block the ability of RASGAPs to stimulate GTP hydrolysis, thus oncogenic RAS is constitutively GTP-bound. HRAS, NRAS, KRAS4A, and KRAS4B (hereafter referred to as KRAS) are ubiquitously expressed in mammalian cells. These RAS isoforms have a near identical G-domain that binds guanine nucleotides and interacts with effector proteins, GTPase activating proteins, and guanine nucleotide exchange factors but have different $\mathrm{C}$ termini and membrane anchors. All RAS isoforms share a common in vitro biochemistry but exhibit different signaling outputs in vivo (Hancock, 2003). Reflecting these differences, each RAS isoform is mutated with different frequencies in different tumors. The major clinical problem is KRAS, which is mutated in $>90 \%$ of pancreatic cancers, $\sim 50 \%$ of colon cancers, and $\sim 25 \%$ of non-small cell lung cancer (Prior et al, 2012).

To generate an output signal, RAS.GTP must recruit effector proteins from the cytosol to the plasma membrane (PM) for activation. One example is the MAPK cascade, where RAS.GTP recruits RAF to the PM for activation, in turn triggering the activation of MEK and ERK. Therefore, RAS proteins must be localized to the PM and correctly arrayed into nanoclusters for biological activity. Nanoclusters are transient RAS-lipid assemblies containing 5-6 RAS proteins that are the sites of effector activation (Murakoshi et al, 2004; Hancock \& Parton, 2005; Plowman et al, 2005; Tian et al, 2007; Zhou \& Hancock, 2015). KRAS is targeted to the PM by a C-terminal membrane anchor that comprises a farnesyl-cysteine-methyl-ester and a polybasic domain (PBD) of six contiguous lysine residues (Hancock et al, 1990). We recently used quantitative spatial imaging analyses and atomistic molecular dynamics to systematically examine the mechanism of association of this KRAS bi-partite PBDprenyl membrane anchor with the PM. Traditionally, PBDs have been thought to interact with PM exclusively via electrostatics where the total number of basic residues determines the strength of electrostatic association with anionic lipids. However, we discovered that the molecular mechanism of KRAS PM binding is considerably more complex. The precise PBD amino acid sequence and prenyl group define a cryptic combinatorial code for lipid ${ }^{1}$ Department of Integrative Biology and Pharmacology, McGovern Medical School, University of Texas Health Science Center, Houston, TX, USA ${ }^{2}$ Department of Diagnostic
and Biomedical Sciences, School of Dentistry, University of Texas Health Science Center, Houston, TX, USA ${ }^{3}$ The University of Texas MD Anderson Cancer Center UTHealth
Graduate School of Biomedical Sciences, Houston, TX, USA

Correspondence: john.f.hancock@uth.tmc.edu 
binding that extends beyond simple electrostatics; within this code, lysine and arginine residues are nonequivalent and prenyl chain length modifies nascent PBD lipid preferences. The code is realized by dynamic tertiary structures on the PM that govern amino acid side chain-lipid interactions and, thus, endow exquisite binding specificity for defined anionic phospholipids (Zhou et al, 2018). An important consequence is the ability of such anchors to sort or retain specific subsets of phospholipids into nanoclusters with a defined lipid composition. In this context, the structure of the KRAS anchor encodes exquisite binding specificity for phosphatidylserine (PtdSer) lipids with one saturated and one desaturated acyl chain (Zhou et al, 2014, 2017; Zhou \& Hancock, 2015). The structure of the KRAS anchor, therefore, renders KRAS PM binding and, hence, KRAS function critically dependent on PM PtdSer content.

Preventing KRAS PM localization has been long advocated as an approach to block oncogenic function. However, early attempts to use farnesyltransferase inhibitors to prevent the first step of posttranslational processing that adds the KRAS membrane anchor failed because KRAS can be alternatively prenylated by geranylgeranyltransferase1 (GGTase1) when cells are treated with farnesyltransferase inhibitors (Hancock, 2003; Sebti \& Der, 2003; Rowinsky, 2006). To identify an alternative strategy, we focused on the dependence of KRAS on PM PtdSer. We showed previously that indirect approaches to reduce PM PtdSer by manipulating sphingolipid and ceramide metabolism was moderately successful in reducing KRAS oncogenesis (Cho, 2016; van der Hoeven et al, 2017). Here, we evaluate direct targeting of the cellular machinery that actively maintains PM PtdSer content. PtdSer is the major anionic lipid on the inner leaflet of the PM comprising 20 mol\% of total lipid content (Vance \& Steenbergen, 2005). The homologs oxysterolrelated binding proteins, ORP5 and ORP8, encoded by OSBPL5 and OSBPL8, respectively, are lipid transport proteins that function at membrane contact sites (MCSs) between the ER, PM, and other organelles. Both proteins transport PtdSer from its site of synthesis in the ER to the PM, where it is exchanged for phosphatidylinositol4-phosphate (PI4P) (Filseck et al, 2015; Sohn et al, 2016). Therefore, we hypothesized that targeting PtdSer transporters ORP5 and ORP8 would disrupt KRAS PM localization and nanoclustering and attenuate KRAS function.

\section{Results}

\section{Knockdown of ORP5 and ORP8 expression inhibits oncogenic KRAS signaling in vivo}

We have shown previously that KRAS PM localization and nanoclustering are critically dependent on the PtdSer content of the inner PM leaflet (Zhou et al, 2014). ORP5 and ORP8 are lipid exchangers involved in the transport of PtdSer from the ER to the PM (Fig 1A); therefore, we hypothesized that inhibition of either protein will deplete the PM of PtdSer and inhibit KRAS signaling through mislocalization of KRAS from the PM. We first investigated whether PtdSer ER to PM transport is relevant for KRAS function in the model organism Caenorhabditis elegans, which expresses a single RAS
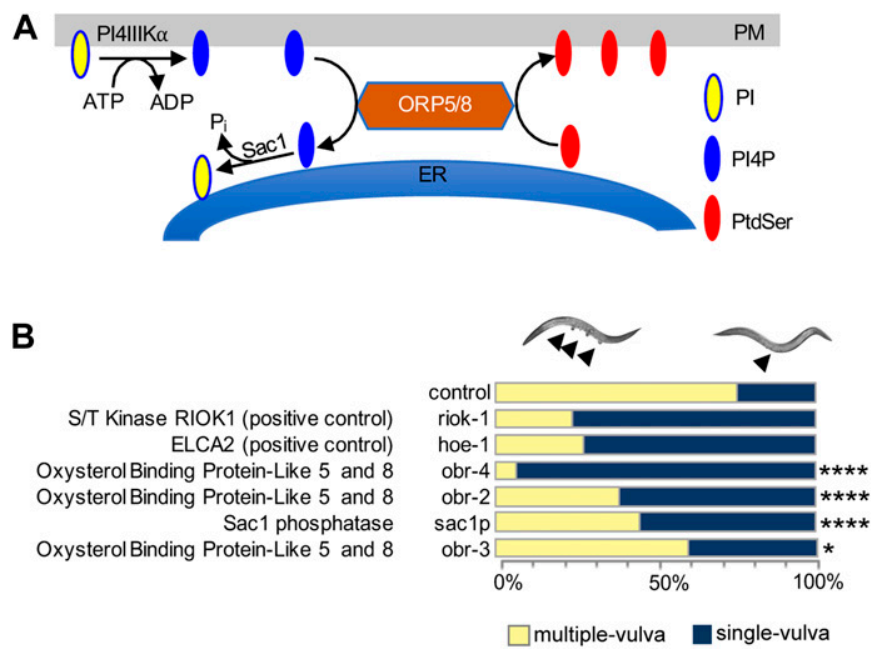

Figure 1. ORP5 and ORP8 transport PtdSer to the PM.

(A) OSBPL5 and OSBPL8 are paralogs that encode ORP5 and ORP8. These proteins are lipid transporters involved in lipid counter transport between the ER and the PM; they specifically exchange PtdSer in the ER with PI4P in the PM. The driving force of this process is a PI4P concentration gradient, whereby PI4P levels are high in the PM and are kept low at the ER by the action of the SAC1 phosphatase which immediately hydrolyzes PI4P. (B) RNAi knockdown screen of OSBPL5, OSBPL8, and SAC1P orthologs in an activated let-60 C. elegans. RNAi was induced by feeding let-60(n1046) L1 worms through adult stage with E. coli strain HT115, producing dsRNA to target genes. The presence of the MUV phenotype was scored using Differential Interface Contrast (DIC)/ Nomarski microscopy. Previous reports show that heo-1 and riok-1 potently suppress the let-60 G13D MUV phenotype and, hence, were used as positive controls ( ${ }^{* \star * *} P<$ $0.0001,{ }^{\star} P<0.05$ ). OSBPL, oxysterol-binding protein like; obr, oxysterol-binding protein related; SAC1P, SAC1-like phosphatidylinositide phosphatase.

gene, let-60, an ortholog of KRAS4B. We performed RNAi-mediated knockdown of validated orthologs of ORP5 and ORP8 in C. elegans carrying a mutationally activated G12D let-60 allele (n1046), whose signaling leads to a multi-vulva (MUV) phenotype that is readily quantifiable. Because there are no clear homologs or orthologs of ORP5 and ORP8 in the worm, we performed blast analysis using the WormBase tool of the National Center for Biotechnology Information (NCBI) OSBPL5 and OSBPL8 sequences and obtained three hits: obr2, obr-3, and obr-4. All three candidates affected the MUV phenotype when tested using RNAi with obr-4, demonstrating the strongest phenotype followed by obr-2 and obr-3 (Table S1). Knockdown of either obr-4 or obr-2 expression potently suppressed the MUV phenotype, with $93 \%$ and $62 \%$ of the population, respectively, displaying a single vulva. This extent of suppression is similar to the positive controls riok-1 and hoe-1, previously described as potent suppressors of the MUV phenotype (Smith \& Levitan, 2004; Weinberg et al, 2014) (Fig 1B). The enzyme Sac1 phosphatase resides in the ER and hydrolyzes PI4P to PI, creating a PI4P concentration gradient where it is high in the PM and low at the ER. This concentration gradient is also the driving force of ORP5/8 function (Filseck et al, 2015). Concordantly, we see that RNAi knockdown of sac1p (human SAC1P) also significantly inhibited the MUV phenotype, however, to a lesser degree than knockdown of ORP5/8. Importantly, viability of organisms was not compromised upon OSBPL5, OSBPL8, or SAC1P gene silencing. Together, these results suggest that ER to PM PtdSer transport is required to support KRAS oncogenic signaling. 


\section{Knockdown of ORP5 and ORP8 expression inhibits PtdSer transport to the PM and mislocalizes KRASG12V from the PM}

To extend the C. elegans observations to mammalian cells, we used CRISPR/Cas9 to knock out (KO) ORP8 in CaCO-2 colorectal cancer cells (Fig 2A). ORP8 KO cells were then transfected with GFP-tagged oncogenic KRAS4B (GFP-KRASG12V) or a GFP-tagged PtdSer probe (GFP-LactC2). Intact basal PM sheets were prepared from these cells, labeled with GFP-antibodies coupled directly to 4.5-nm gold particles and visualized by EM (Hancock and Prior, 2005). We observed a significant decrease in anti-GFP immunogold labeling of both KRASG12V (Figs 2B and S1A) and LactC2 (Figs 2C and S1B) in ORP8 KO cells, indicating mislocalization from the inner PM. Spatial mapping analysis showed that the extent of clustering ( $L$ max) of KRASG12V remaining on the PM was also significantly decreased upon loss of ORP8 expression. Concordant with reduced KRASG12V $\mathrm{PM}$ binding and nanoclustering loss of ORP8 expression resulted in decreased MAPK signaling as measured by ppERK output (Fig 2A). To validate the mechanistic consequences of ORP8 $\mathrm{KO}$, we measured the PM levels of PI4P, PIP , and $\mathrm{PIP}_{3}$. To that end, we transfected CaCO-2 cells with GFP-tagged lipid probes and examined the extent of anti-GFP immunogold labeling by EM of intact PM sheets. The GFP-FAPP1-PH probe (FAPP1-PH) contains the pleckstrin homology (PH) domain of the FAPP1 protein, which selectively binds PI4P (Balla et al, 2005). The GFP-PLC $\delta$-PH probe (PLC $\delta-P H)$ comprises the $\mathrm{PH}$ domain of PLCS, which selectively binds $\mathrm{PIP}_{2}$ (Hammond et al, 2012). The GFP-AKT-PH probe contains the PH domain of AKT which binds $\mathrm{PIP}_{3}$ (Miao et al, 2010). In these EM experiments, we observed a significant increase in the amount of PI4P on the PM in ORP8 KO cells, which was further validated with GFP-P4M-SidM (Hammond et al, 2014), a second PI4P probe that exhibits a higher affinity for PI4P and has been shown to better detect P4P on the PM (Figs 2D and E and S1C and D). Elevated PM levels of PI4P also correlated with significantly increased $\mathrm{PM}$ levels of both $\mathrm{PIP}_{2}$ (Figs $2 \mathrm{~F}$ and $\mathrm{S} 1 \mathrm{E}$ ) and $\mathrm{PIP}_{3}$ (Figs $2 \mathrm{G}$ and S1F).

CaCO-2 cells do not form a well-organized confluent monolayer and are, thus, not well-suited for quantitative confocal microscopy analysis. We, therefore, used confluent MCF-7 breast cancer cells to visualize and quantify KRAS and PtdSer mislocalization. ORP5 and ORP8 were knocked down separately as well as simultaneously in MCF-7 cells (Fig 3A). Parental and knockdown cells were infected with bicistronic lentiviruses expressing either GFP-KRASG12V or GFP-LactC2 with mCherry-CAAX, a general endomembrane marker, and analyzed by confocal microscopy (Fig 3B). The extent of overlap between GFP and mCherry signals, indicative of colocalization, was quantified by Manders coefficients. The higher the Manders coefficient, the more extensive the colocalization of KRASG12V or LactC2 with endomembranes. These experiments showed that knockdown of ORP5 or ORP8 individually mislocalized KRASG12V and LactC2 from the PM to endomembranes to similar extents, with no discernible additive effect in double knockdown cells (Fig 3C).

\section{Depletion of PM PtdSer inhibits cell proliferation and anchorage-independent growth}

We next evaluated the effects of ORP8 knockdown on KRAS and PtdSer localization in a panel of pancreatic cancer cell lines that are wild-type for KRAS, such as BXPC-3, or contain a KRAS mutation, such as PANC-1, MiaPaCa-2, and $\mathrm{MOH}$. For each cell line, multiple stable monoclonal ORP8 knockdown (KD) cells were generated using shRNA lentiviral infection followed by puromycin selection. The use of multiple clones was designed to examine potential clonal variation after cell line selection. As in the model MCF-7 cell line, ORP8 knockdown caused significant mislocalization of both
A

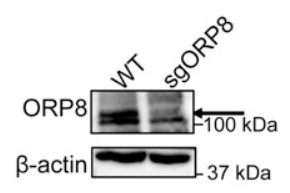

B
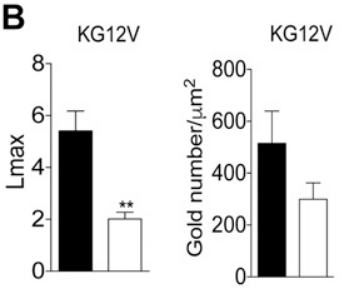

E

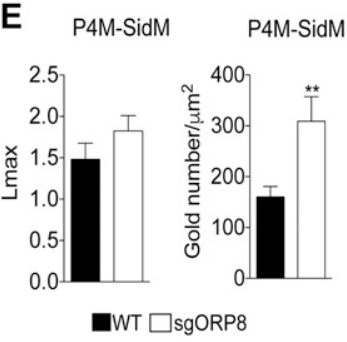

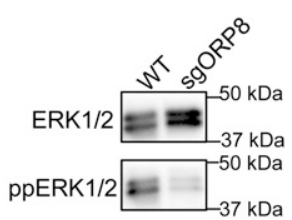

C
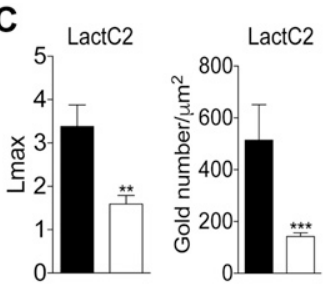

$\mathbf{F}$
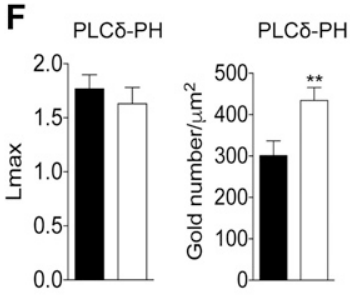

D
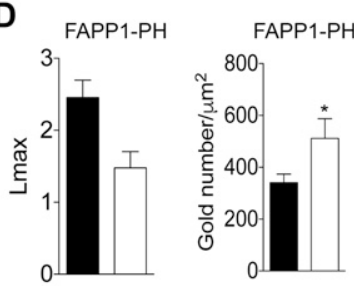

\begin{abstract}
G $\quad$ AKT-PH
\end{abstract}
Figure 2. KRAS and PtdSer, PI4P, $\mathrm{PIP}_{2}$, and $\mathrm{PIP}_{3}$ clustering and membrane localization changes in OSBPL8 CRISPR KO CaCo-2 cells.

(A, B, C, D, E, F, G) CRISPR/Cas9 KO of ORP8 in CaCO-2 cells was validated by Western blotting and MAPK signaling assayed as ppERK levels in Western blots. Total ERK and $\beta$-actin levels were used as loading controls. PM sheets prepared from CaCO-2 parental (WT) and SgORP8 cells transiently transfected with GFP-KRASG12V (B), GFP-LactC2 (C), GFP-FAPP1-PH (D), GFP-P4M-SidM (E), GFP-PLC 8 -PH (F), or GFP-AKT-PH (G) were labeled with anti-GFP antibodies coupled directly to 4.5-nm gold particles and visualized by EM. The amount of KRASG12V, LactC2, FAPP1-PH, P4MSidM, PLC $-\mathrm{PH}$, and AKT-PH on the PM was measured as gold particle labeling per $\mu \mathrm{m}^{2}$, and significant differences were quantified using $t$ tests. Clustering of the GFP-tagged probes were quantified by univariate spatial analysis, summarized as Lmax values and significant differences were assessed using bootstrap tests $( \pm$ SEM,$n \geq 12)\left({ }^{*} P<0.05,{ }^{* *} P<0.01\right.$, ${ }^{* * *} P<$ $0.001,{ }^{* * * *} P<0.00001$; KG12V: KRASG12V, LactC2: PtdSer probe, FAPP1-PH and P4M-SidM: PI4P probes, PLCס$\mathrm{PH}$ : PIP 2 probe, AKT-PH: PIP3 probe). 


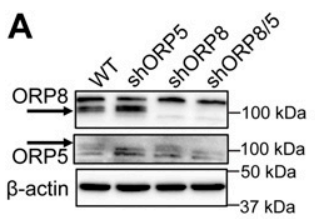

c
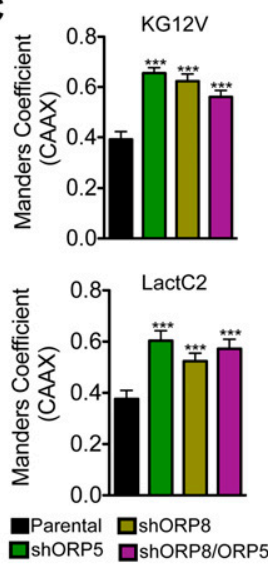

B

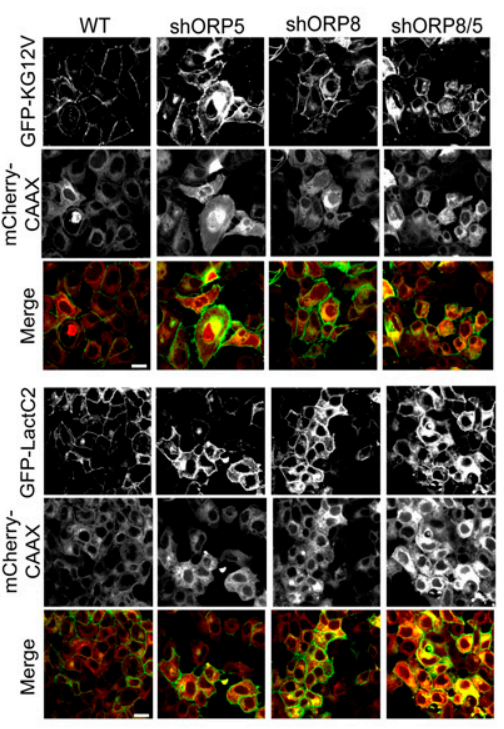

Figure 3. Knockdown of ORP5 or ORP8 mislocalizes KRAS and PtdSer from the PM.

(A) shRNA knockdown of ORP5 and ORP8 separately and simultaneously in MCF-7 breast cancer cells was validated by Western blotting, and $\beta$-actin levels were used as loading controls. (B) Parental (WT) and knockdown cells were transiently transfected with GFP-KRASG12V and mCherryCAAX (an endomembrane marker) or GFP-LactC2 and mCherryCAAX and imaged in a confocal microscope. Representative images are shown. (C) The extent of KRAS and LactC2 mislocalization was quantified using Manders coefficient, which measures the extent of colocalization/overlap of GFP and mCherry signals. Significant differences were evaluated using $t$ tests $( \pm$ SEM, $n \geq 6)\left({ }^{*} P<0.05\right.$, ${ }^{* *} P<$ $0.01,{ }^{* * *} P<0.001$ ); scale bar $20 \mu \mathrm{m}$.

KRASG12V and LactC2 from the PM in each cell line tested, with the accumulation of both probes on endomembranes as visualized by confocal microscopy (Fig S2). We also generated monoclonal ORP5 knockdown cells using a similar protocol, as well as double knockdown of both ORP5 and ORP8 to assess possible compensation by one ORP homolog in the absence of the other (Fig S3).

First, we tested the effects on cell proliferation over the course of $5 \mathrm{~d}$ (Fig 4). In the case of the KRAS WT cell line BxPC-3, there was no discernible effect of knockdown of either ORP5 or ORP8 alone on proliferation rate. However, the simultaneous knockdown of both proteins modestly decreased the proliferation rate. In the case of PANC-1, which is a KRAS-independent cell line, that is, it is not addicted to oncogenic KRAS (Singh et al, 2009), ORP5 or ORP8 single knockdown resulted in cells growing significantly faster than parental cells; however, double knockdown clones grew more slowly. In contrast, single gene knockdown of either ORP homologue was sufficient to inhibit the proliferation of KRAS-dependent MiaPaCa-2 and $\mathrm{MOH}$ cells. In these proliferation assays, all $\mathrm{MOH}$ clones tested displayed a consistent response (Fig 4), whereas there was some variation in the behavior of different MiaPaCa2 ORP8 knockdown clones, including some that underwent senescence in culture and could not be analyzed further.

Anchorage-independent growth is a more stringent assessment of tumorigenic potential; therefore, we analyzed colony formation in soft agar (Fig 5). MiaPaCa-2 ORP5 and ORP8 KD clones showed

BxPC-3
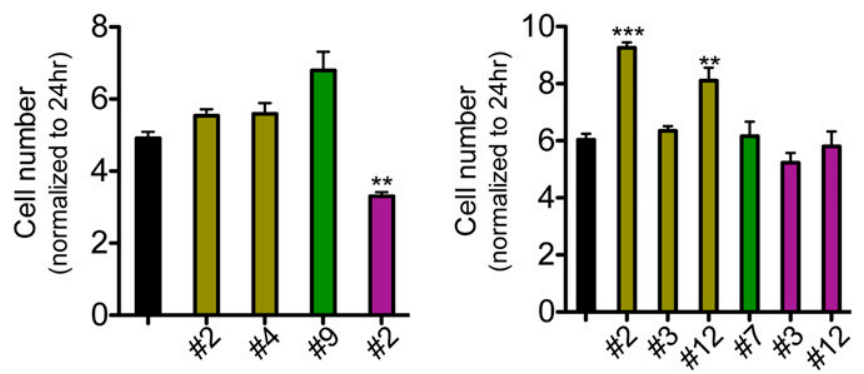

MiaPaCa-2

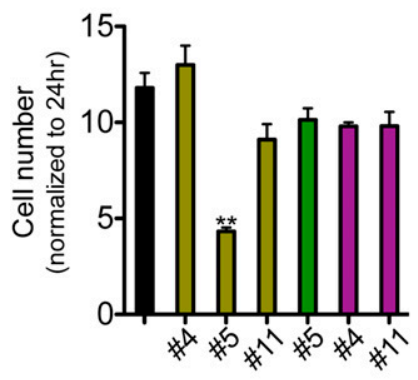

Parental

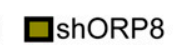

Figure 4. ORP5 or ORP8 knockdown decreases the growth rate of KRASdependent pancreatic cancer cells

ORP5 and ORP8 were knocked down separately and simultaneously by shRNA in BXPC-3, PANC-1, MiaPaCa-2, and MOH cells. Parental and knockdown cells were grown in six-well plates for $5 \mathrm{~d}$ and counted every day. Cell numbers of each cell line at day 5 were normalized to their cell number at $24 \mathrm{~h}$ and plotted. Significant differences were evaluated using $t$ tests $( \pm$ SEM, $n=3)\left({ }^{* *} P<0.01,{ }^{* * *} P<0.001\right)$.

heterogeneous responses that correlated with their proliferation rate; MiaPaCa-2 KD clones that grew slower also showed reduced growth in soft agar. Double knockdown of both ORP proteins, however, had a more significant effect on anchorage-independent growth than on proliferation. Knockdown of either ORP protein completely abrogated colony formation in $\mathrm{MOH}$ cells. Interestingly, the clonal variation in MiaPaCa-2 ORP8 KD cells also correlated with the extent of mislocalization of LactC2 and KRASG12V as determined by confocal microscopy (Fig S4). Conversely, knockdown of ORP8 alone had no significant effect on the anchorage-independent growth of KRAS-independent PANC-1 cells. Of note, PANC-1 cells were more sensitive to knockdown of ORP5 than ORP8, whereas ORP8 knockdown more potently affected MiaPaCa-2 and $\mathrm{MOH}$ cells. As in the proliferation assays, double knockdown of both homologs had a stronger effect in all three KRAS-mutant transformed cell lines.

Analysis of signaling pathways revealed a paradoxical increase in MAPK signaling in nearly all KRAS-mutant ORP5 and ORP8 KD clones, evidenced as elevated ppERK1/2 levels. This likely indicates alleviation of the negative feedback on upstream components of the RAF-MEK-ERK pathway, in turn reflecting a reduction in the strength of KRAS signaling. One exception was PANC-1 KD cells which showed increased ppERK levels only when ORP5 was individually knocked down consistent with better response of these cells to ORP5 KD. Levels of pThr308AKT were increased in MiaPaCa-2 knockdown cells compared with parental and empty vector 
BxPC-3

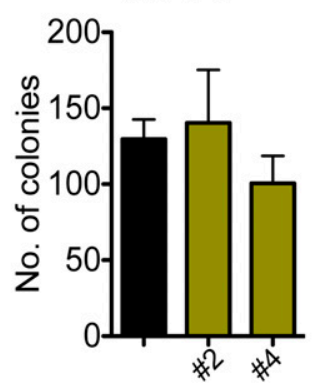

MiaPaCa-2

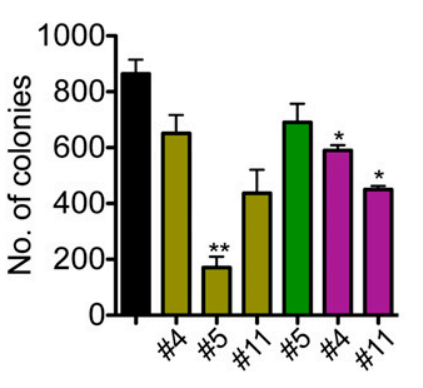

Parental $\square$ shORP8

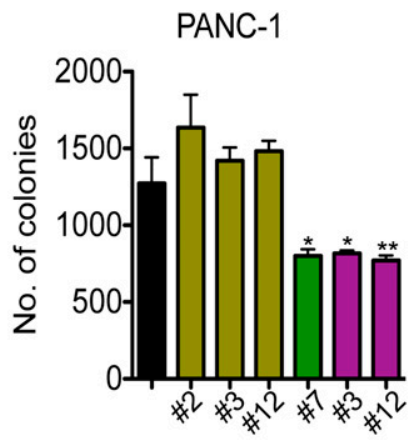

$\mathrm{MOH}$

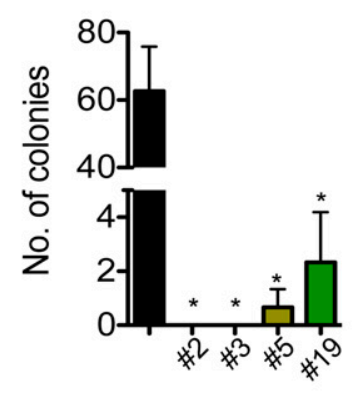

shORP5 口shORP8/ORP5

Figure 5. ORP5 or ORP8 knockdown decrease anchorage-independent growth capability of KRAS-dependent pancreatic cancer cells.

ORP5 and ORP8 were knocked down separately and simultaneously by shRNA in BXPC-3, PANC-1, MiaPaCa-2, and MOH cells. Parental and knockdown cells were seeded in soft agar, with a base layer of $1 \%$ agar-media mixture and a top layer of a $0.6 \%$ agar-cell suspension mix in six-well plates. After $2-3 w k$, the colonies were stained with $0.01 \%$ crystal violet and imaged. Colony numbers were quantified by Image and significant differences were evaluated using $t$ tests $( \pm$ SEM, $n=3)\left({ }^{\star} P<0.05,{ }^{* *} P<0.01\right)$.

controls, presumably because of the accumulation of PI4P and, hence, $\mathrm{PIP}_{2}$ on the PM after ORP knockdown (Fig 6), feeding into the PI3K/AKT pathway. In contrast, no pThr308AKT was detected in either parental or knockdown $\mathrm{MOH}$ cells. There was no significant difference in pThr308AKT levels between parental and knockdown cells in the PANC-1 and BxPC-3 lines. These results may possibly reflect different signaling outputs from the different KRAS point mutations in the cell lines (MiaPaCa-2: G12C, MOH: G12R, PANC-1: G12D) as well as KRAS dependency. pSer473AKT levels were elevated in KRAS-mutant ORP5 and ORP8 KD cells in all cell lines tested. Increased levels of YAP-1 and Epidermal Growth Factor Receptor (EGFR) activation have been previously reported to be compensatory mechanisms to KRAS inhibition in pancreatic cancer cells (Vartanian et al, 2013; Kapoor et al, 2014). Across the panel of KD cells, we saw considerable clonal variation with regard to YAP-1 and pEGFR levels (Fig 6) with no obvious correlation with KRAS mutational status. Finally, to evaluate whether KRAS mutational status affects ORP5 and ORP8 basal levels, we used the isogenic colorectal cancer cell line HCT116; the parental line harbors a heterozygous KRAS mutation, whereas its derivative line has a single WT KRAS allele after KO of the mutant allele via homologous recombination (Markowitz et al, 2009). ORP8 levels increased primarily in the derivative line upon ORP5 knockdown; however, the reverse was not observed (Fig S5).

Inhibiting class III PI4K $\alpha$ mislocalizes PtdSer and KRAS from the PM and selectively inhibits proliferation of KRAS-mutant pancreatic cancer cells

Currently, there are no available ORP5 or ORP8 inhibitors; therefore, we targeted the upstream component of PtdSer exchange: PI4KIII $\alpha$. Class III $\mathrm{PI} 4 \mathrm{~K} \alpha$ converts phosphatidylinositol (PI) to PI4P at the PM, which is then exchanged for PtdSer from the ER via ORP5 and ORP8 (Nakatsu et al, 2012; Clayton et al, 2013). The driving force of this process is a PI4P concentration gradient, which is kept high at the PM and low at the ER by Sac1 phosphatase which hydrolyzes PI4P back to PI (Fig 1A). The selective class III PI4K $\alpha$ inhibitor compound 7 (C7) (Waring et al, 2014; Boura \& Nencka, 2015; Raubo et al, 2015) should dissipate the PI4P concentration gradient between the PM and ER, functionally inhibiting ORP5 and ORP8. Because PI4KIII $\alpha$ provides the driving force for both ORP5 and ORP8, inhibition should phenocopy a knockdown of both homologs.

MDCK cells stably expressing GFP-KRASG12V and mCherry-CAAX, or GFP-LactC2 and mCherry-CAAX were treated with C7 for $48 \mathrm{~h}$ and analyzed by confocal microscopy. Treatment with the inhibitor potently mislocalized both LactC2 and KRASG12V in a dosedependent manner, with significant mislocalization seen at $30 \mu \mathrm{M}$ (Fig 7A and B), consistent with the previously reported concentrations of $\mathrm{C} 7$ required to reduce cellular $\mathrm{PIP}_{2}$ and $\mathrm{PIP}_{3}$ levels $\left(I C_{50}=30 \mu \mathrm{M}\right)$ (Waring et al, 2014). To further quantify the amount of KRASG12V and LactC2 on the PM as well as the extent of nanoclustering after drug treatment, intact basal PM sheets of MDCK cells were labeled with gold-conjugated anti-GFP antibodies $48 \mathrm{~h}$ after treatment with $30 \mu \mathrm{M}$ of $\mathrm{C} 7$ and analyzed by EM. C7 treatment caused significant mislocalization of both KRASG12V and LactC2 from the PM and decreased their nanoclustering (Fig 7C).

Finally, we tested the effects of $\mathrm{C} 7$ on cell proliferation in a panel of pancreatic cancer cell lines as well as the immortalized pancreatic cell line HPNE. Ten different concentrations of $\mathrm{C} 7$ were tested ranging from $1 \mathrm{nM}$ to $30 \mu \mathrm{M}$. The results show that $\mathrm{C} 7$ had no effect on the nontransformed cell line HPNE. All transformed cells were sensitive to C7, but the calculated $\mathrm{IC}_{50}$ values for growth inhibition were much lower for KRAS mutant than KRAS WT cells, with the most sensitive lines being the KRAS-dependent $\mathrm{MOH}$ and MiaPaCa-2 lines (Fig 7D).

\section{Expression of ORP5 and ORP8 in KRAS-mutant cancers}

High ORP5 expression has been previously shown to be associated with poorer survival rates in patients with pancreatic adenocarcinoma (PDAC) (Koga et al, 2008). To further investigate whether this is linked to oncogenic KRAS signaling, we analyzed OSBPL5 mRNA expression and KRAS mutation data in GDC (Genomic Data Commons) TCGA-PAAD, TCGA-LUNG, and TCGA-PANCAN, cohorts of pancreatic adenocarcinoma, non-small cell lung cancer, and a pan-cancer cohort comprising 33 different cancer types, respectively (Goldman et al, 2019 Preprint). In all three cohorts, ORP5 expression was significantly up-regulated in KRAS-mutant subgroups compared with KRAS wild-type subgroups (Fig 8A). Additional analyses showed that increased expression of ORP5 or ORP8 correlate with shorter overall survival times for patients in all three cohorts (Fig 8B and C). 


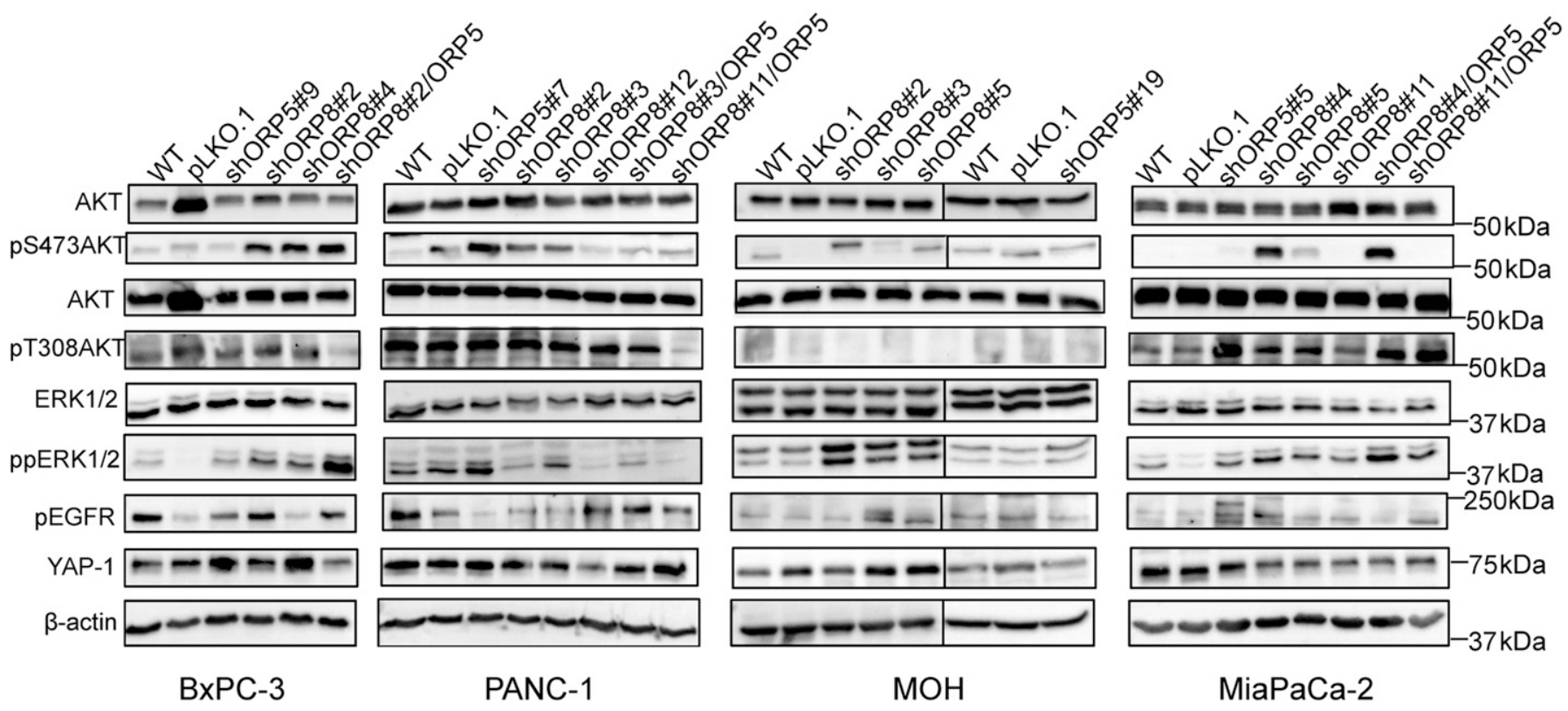

Figure 6. Consequences of ORP5 and ORP8 knockdown on downstream MAPK and PI3K/AKT signaling.

Protein from BXPC-3, PANC-1, MiaPaCa-2, and MOH parental, single and double ORP knockdowns as well as cells transfected with empty vector control (pLKO.1) were harvested, and $20 \mu \mathrm{g}$ was subjected to SDS-PAGE and used for Western blotting. EGFR, MAPK, and PI3K signaling were assayed as pEGFR, ppERK, and pAKT levels, respectively. Amplification of YAP-1 was also evaluated. Total ERK, total AKT, and $\beta$-actin levels were used as loading controls.

\section{Discussion}

We show here that maintenance of PM PtdSer levels is absolutely required to maintain KRAS PM localization and hence oncogenic function. Thus, knockdown or inhibition of any component of the ORP5/ 8 ER to PM PtdSer transport process abrogates KRAS function in multiple cells and organisms. First, RNAi silencing of orthologs of
ORP5, ORP8, or the ER PI4P phosphatase, Sac1, inhibited oncogenic let-60 (KRAS) signaling in C. elegans. Second, shRNA knockdown or CRISPR/Cas9 KO of ORP5 or ORP8 mislocalized PtdSer and KRAS from the PM and decreased the extent of KRAS PM clustering in human pancreatic, breast and colorectal cancer cells. ORP5 or ORP8 knockdown concordantly inhibited the proliferation and anchorage-independent growth of KRAS-dependent pancreatic
A

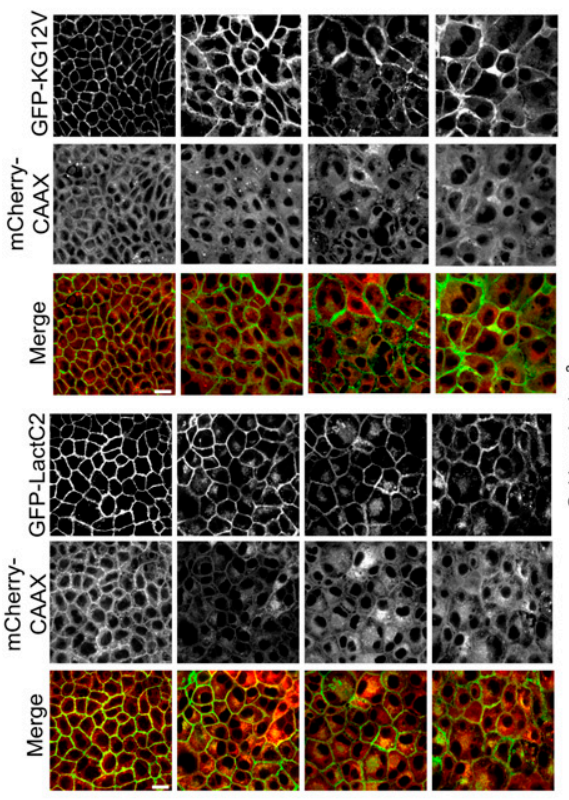

B

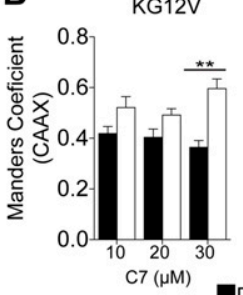

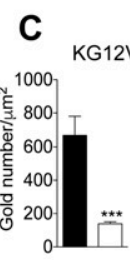

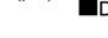

LactC2

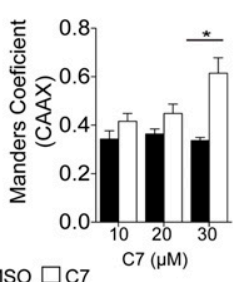

LactC2

D

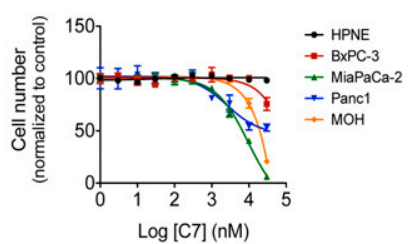

Figure 7. PI4KIII $\alpha$ inhibition mislocalizes KRAS and PtdSer from PM and inhibits growth of KRASdependent pancreatic cancer cells.

(A) MDCK cells stability expressing GFP-KG12V and mCherryCAAX or GFP-LactC2 and mCherryCAAX were treated with DMSO or the PI4KIII $\alpha$ inhibitor $\mathrm{C7}$ for $72 \mathrm{~h}$ at varying concentrations, and then imaged with confocal microscopy. Representative images are shown. (B, C) The extent of KRAS and LactC2 mislocalization was quantified using Manders coefficient, which measures the extent of colocalization/overlap of GFP and mCherry signals. Significant differences were quantified using $t$ tests $( \pm S E M, n \geq 5)$ (C) Basal PM sheets from MDCK cells in (A) treated with $30 \mu \mathrm{M}$ of $\mathrm{C} 7$ for $48 \mathrm{~h}$ were prepared and labeled with anti-GFP antibodies coupled directly to 4.5$\mathrm{nm}$ gold particles and visualized by EM. The amount of KRASG12V and LactC2 on the PM was measured as gold particle labeling per $\mu \mathrm{m}^{2}$, and significant differences were quantified using $t$ tests. KRAS and LactC2 clustering were quantified by univariate spatial analysis, summarized as $L$ max values and significant differences were assessed using bootstrap tests ( \pm SEM, $n \geq 12$ ). (D) HPNE, BXPC-3, MiaPaCa-2, PANC-1 and MOH cells were seeded in 96-well plates. After $24 \mathrm{~h}$, fresh growth medium supplemented with $1 \%$ DMSO or increasing C7 concentrations were added and the cells were allowed to grow for another $72 \mathrm{~h}$ and then counted $( \pm S E M, n=3)$ $\left({ }^{\star} P<0.05,{ }^{\star \star} P<0.01,{ }^{\star \star \star} P<0.001 ;\right.$ KG12V: KRASG12V, LactC2: PtdSer probe), scale bar $20 \mu \mathrm{m}$. 

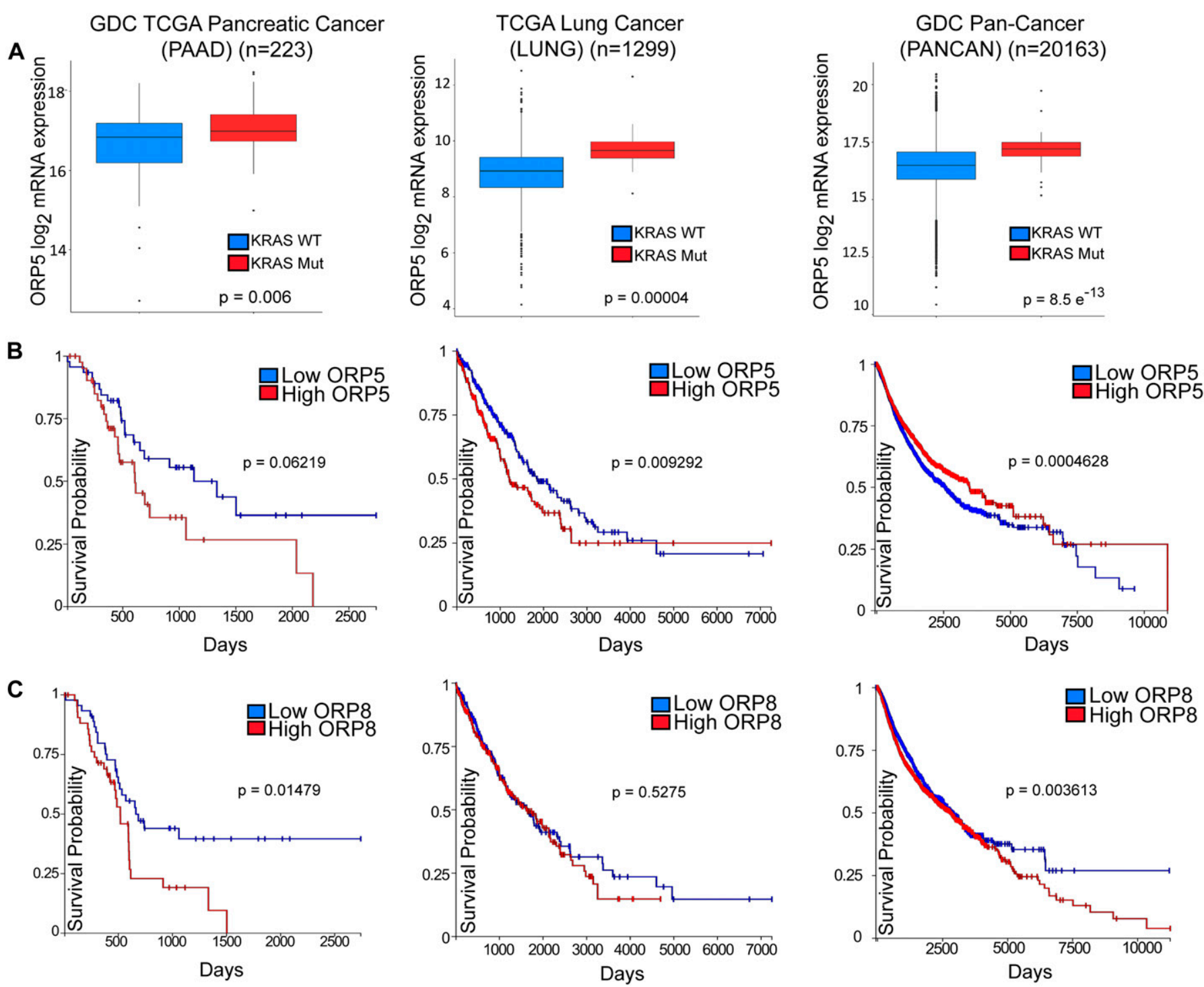

Figure 8. High expression levels of OSBPL5 and OSBPL8 correlate with poorer prognosis in cancer patients.

$(A, B, C)$ Box plots indicating quartiles of ORP5 mRNA expression level in patient samples in cohorts of pancreatic cancer (GDC TCGA PAAD, $n=223$ ), lung cancer (TCGA LUNG, $\mathrm{n}=1,299$ ), and of 33 types of cancer (GDC Pan-Cancer [PANCAN], $\mathrm{n}=20,163$ ) with or without KRAS mutations. Statistical significance was analyzed with Welch's $t$ test. Kaplan-Meier survival plots based on expression levels of ORP5 (B) and ORP8 (C) in cohorts listed in (A). Plots were generated using the University of California, Santa Cruz (UCSC) Xena Browser.

cells. Third, inhibiting PI4KIII a also reduced PtdSer and KRAS PM levels sufficiently to selectively abrogate the growth of KRASdependent pancreatic cancer cells. Finally, these observations have some clinical correlates in that PDAC patients with higher expression levels of ORP5 or ORP8 have poorer clinical outcomes, and more generally, KRAS mutational status is associated with higher ORP5 expression across multiple cancer types. These clinical correlates are consistent with selection for more robust maintenance of PM PtdSer levels to support KRAS oncogenesis.

Recent work suggests that ORP5 is primarily responsible for PtdSer and PI4P trafficking at ER-PM MCSs and that ORP8 may only be recruited to these sites upon $\mathrm{PIP}_{2}$ accumulation. Under conditions of $\mathrm{PIP}_{2}$ accumulation, ORP8 can also exchange $\mathrm{PIP}_{2}$ for PtdSer (Sohn et al, 2018). Therefore, knocking down ORP5 would lead to an accumulation of PI4P at the PM and, hence, an increase in
$\mathrm{PIP}_{2}$ levels, which would lead to ORP8 recruitment to the PM and at least partial restoration of PtdSer PM levels by the dual action of ORP8 as a PtdSer/PI4P and PtdSer/PIP 2 exchanger (Ghai et al, 2017). This would explain why we did not observe an increase of ORP5 in ORP8 HCT116 knockdown cells but observed an increase of ORP8 when ORP5 was knocked down (Sohn et al, 2018). Whether PIP 2 is more important than PI4P for PtdSer transport to the PM and cannot be compensated for remains to be determined. However, the importance of each homolog seems to be cell line specific, as we noticed that PANC-1 cells were more sensitive to ORP5 knockdown, whereas MiaPaCa-2 cells were more sensitive to ORP8 knockdown. ORP5/8 has been reported to also function at ERmitochondria MCSs, and knockdown of either ORP leads to altered mitochondrial morphology and reduced oxygen consumption (Galmes et al, 2016). However, it appears that ORP5 interacts more 
extensively with mitochondrial outer membrane proteins, so it might play a more important role than ORP8 here. It has also been reported that KRAS-independent lines (Ribosomal S6 Kinase [RSK]dependent) depend on oxidative phosphorylation and have increased reactive oxygen species, whereas KRAS-dependent lines rely on glycolysis (Galmes et al, 2016; Yuan et al, 2018). Together, this may be a possible explanation for the increased sensitivity of PANC1 cells to ORP5 knockdown. Nonetheless, all cell lines were more sensitive to the simultaneous knockdown of both ORP5 and ORP8 compared with either one alone. One potential method to partially sustain PtdSer levels on the PM in double knockdown cells may be through increased vesicular transport of PtdSer through recycling endosomes, which are enriched with this phospholipid (Matsudaira et al, 2017). In a recent article, Venditti et al (2019) showed that ORP10 transfers PtdSer from its site of synthesis at the ER to the trans-Golgi (TGN) and that TGN PtdSer levels correspond to the activity levels of phosphatidylserine synthase I in the ER (Venditti et al, 2019). They also showed that ORP10 knockdown resulted in significant reduction of Golgi PtdSer levels, but no change in PM PtdSer levels. To this end, we would speculate that the Golgi pool of PtdSer does not contribute, at least in a noticeable way, to the PM levels of PtdSer and, hence, of KRAS. However, we still do see PtdSer and KRAS located on the PM of ORP5/ORP8 double knockdown MCF-7 cells as well as in C7-treated MDCK cells. Thus, PtdSer that accumulates in the ER because of ORP5/ 8 inactivation may be shuttled to the TGN via ORP10 and transported to the PM via vesicular transport. However, because the PM levels of KRAS and PtdSer after ORP5/8 depletion are still significantly lower than in parental cells, we would again conclude that the Golgi pool of PtdSer is not a major contributor to KRAS PM localization. Further investigation into how inhibition of ORP5 or ORP8 affects vesicular transport is warranted.

Sohn et al (2018) also reported that PI4KIII $\alpha$ inhibition decreased the amount of ORP5 and ORP8 localized to the PM (Sohn et al, 2018). In addition, they showed that prolonged overexpression of PIP5K1b, which converts PI4P to $\mathrm{PIP}_{2}$, caused a redistribution of PI4P to endosomal and Golgi compartments, effectively reducing PI4P PM levels and, therefore, PIP 2 PM levels by limiting substrate availability. This in turn led to a reduction of PtdSer levels on the PM, further validating the rationale of targeting PI4KIII $\alpha$ to reduce PI4P, and hence PtdSer PM levels to inhibit KRAS signaling. Concordantly, we showed that treating MDCK cells with the selective class III PI4K $\alpha$ inhibitor, C7, resulted in redistribution of PtdSer, and hence KRAS, from the PM to endomembranes in a dose-dependent manner. Our results are also in accordance with others who found a $50 \%$ reduction in overall PtdSer levels and a depletion of PtdSer levels at the PM in response to PI4KIII $\alpha$ inhibition or PI4KIII $\alpha$ KO (Chung et al, 2015; Sohn et al, 2016). The decrease of PtdSer synthesis was due to the accumulation of PtdSer at the ER, and its consequent negative feedback inhibition on PtdSer synthase I and II.

Importantly, we also found that $\mathrm{C7}$ selectively inhibited the proliferation of pancreatic cancer cell lines, with KRAS-dependent lines displaying increased sensitivity compared with KRASindependent lines. KRAS wild-type cells were only affected at high concentrations and immortalized normal pancreatic cells were unaffected even at the highest concentrations tested. Previously, PI4KIII $\alpha$ inhibition was found to increase radiosensitivity in diverse cancer cell lines in vitro as well as in immune-competent and nude mouse models of breast and brain cancer (Park et al, 2017). Thus, targeting PI4KIII $\alpha$ is tolerated in these animals at concentrations that result in antitumor effects. Furthermore, there are no reports of activating mutations or deletions of PI4Ks in cancer, allowing us to be cautiously optimistic of a low mutability rate of this gene (Clayton et al, 2013). Finally, PI4KIII $\alpha$ inhibition decreased PAKT levels by decreasing $\mathrm{PIP}_{3}$ PM levels in breast cancer cells; hence, it can act in KRAS-mutant cells as a target to simultaneously inhibit both MAPK and PI3K pathways, which is an attractive notion given that $93 \%$ of PIK3CA mutations in PDAC co-occur with KRAS mutations (Park et al, 2017; Waters \& Der, 2018). Therefore, PI4KIII $\alpha$ has merit as a novel treatment target for KRAS-dependent tumors that warrants further research.

Koga et al (2008) showed that PDAC patients with high ORP5 expression had a $36.4 \%$ 1-yr survival rate, whereas those with low ORP5 expression had a one-year survival rate of $73.9 \%$. In addition, they showed that siRNA knockdown of ORP5 decreased the invasion potential of pancreatic cancer cells in matrigel, whereas overexpression of ORP5 increased invasion. This correlated in patients, whereby high ORP5 expression corresponded with invasion of cancer cells to the main pancreatic duct, leading to early relapse (Koga et al, 2008; Ishikawa et al, 2010). Through analyses of other patient cohorts in the TCGA (The Cancer Genome Atlas) database, we found that this negative correlation between ORP5 expression level and overall survival held true not only in PDAC but also in multiple other cancer types. We also observed significantly increased ORP5 expression in KRAS-mutant tumors compared with KRAS wild-type tumors. We also found that high expression of ORP8 also correlated with decreased patient survival in multiple cancers including PDAC.

In conclusion, we have shown that targeting ORP5, ORP8, or PI4KIII $\alpha$ depletes the PM of PtdSer resulting in mislocalization of KRAS and reduced nanoclustering of KRAS that remains PM bound in all cell lines tested. In turn, these perturbations of KRAS PM interactions lead to reduced proliferative and tumorigenic capacity of KRAS-mutant cancer cells. To the best of our knowledge, this is the first study to establish the mechanistic connection between ORP5 and ORP8 with KRAS and their important role in KRAS-driven cancers. Our results with ORP5 and ORP8 knockdown as well as with PI4KIIl $\alpha$ inhibition have possible implications for cancer therapy in KRAS-mutant tumors. In sum, we have demonstrated that reducing PM PtdSer levels is selectively toxic to KRAS-transformed cells and that there was no organismal toxicity associated with blocking PtdSer ER to PM transport in C. elegans, whereas activated let-60 signaling was suppressed. The exquisite binding specificity of the KRAS membrane anchor for PtdSer, which is essential for PM targeting, is thus a vulnerability in KRAS-mutant tumors that may be amenable to therapeutic exploitation.

\section{Materials and Methods}

\section{Materials}

Class III PI4K alpha inhibitor Small Molecule (Tool Compound), C7, was purchased from Cancer Research UK (ximbio.com, cat. no. 153579, distributed by Ximbio) and dissolved in DMSO. Cell culture 
media were purchased from HyClone and GIBCO. FBS was purchased from GIBCO. Puromycin was purchased from Thermo Fisher Scientific (BP2956-100). Anti-phospho-p44/42 MAPK (ERK1/2) Thr202/Tyr204 (43702), total ERK1/2 (4659S), p-c-Raf (9427S), phospho-Ser473 AKT (4060L), phospho-Thr308 AKT (9257S) pan-AKT (2920S), phospho-EGFR (4407L), GFP (2956S), and $\beta$-Actin (A1978) antibodies were purchased from Cell Signaling Technology. Antiosbpl5 (NB100-57071) and YAP-1 (NB110-58358) antibodies were purchased from Novus. Anti-osbpl8 (ab99069) antibody was purchased from Abcam. Rabbit anti-mGFP antibodies for immunogold labeling were generated in house. Agarose-low melting point (CAS 39346-81-1) was purchased from Sigma-Aldrich.

\section{Cell lines}

MDCK, HPNE, and PANC-1 cells were purchased from American Type Culture Collection. TLA293T cells were a generous gift from Dr Guangwei Du, McGovern Medical School, Houston, TX. BxPC3, MOH, and MiaPaCa-2 were kindly provided by $\mathrm{Dr}$ Craig Logsdon at MD Anderson Cancer, Houston, TX. KRAS (+/-) HCT116 isogenic cell line pair was kindly provided by Dr Scott Kopetz at MD Anderson Cancer Center, Houston, TX. MDCK, PANC-1, and TLA293T cells were grown in DMEM supplemented with $2 \mathrm{mM}$ L-glutamine and 10\% FBS. HPNE cells were cultured in 75\% DMEM and 25\% Medium M3 Base supplemented with $5 \% \mathrm{FBS}, 10 \mathrm{ng} / \mathrm{ml}$ human recombinant EGF, $5.5 \mathrm{mM}$ D-glucose, and $750 \mathrm{ng} / \mathrm{ml}$ puromycin. MiaPaCa-2 cells were cultured in DMEM supplemented with $2 \mathrm{mM} \mathrm{L-glutamine}$ and 10\% FBS and $2.5 \%$ horse serum. BxPC3 and MOH cells were cultured in RPMI-1640 medium supplemented with $2 \mathrm{mM} \mathrm{L-glutamine}$ and 10\% FBS. HCT116 cells were cultured in RPMI 1640 including 2 mM L-glutamine and 25 $\mathrm{mM}$ sodium bicarbonate, supplemented with $10 \% \mathrm{FBS}$. All cell lines were grown at $37^{\circ} \mathrm{C}$ in $5 \% \mathrm{CO}_{2}$.

\section{Western blotting}

Cells were washed in cold PBS and lysed in buffer containing $50 \mathrm{mM}$ Tris- $\mathrm{Cl}$ (pH 7.5), $75 \mathrm{mM} \mathrm{NaCl}, 25 \mathrm{mM} \mathrm{NaF}, 5 \mathrm{mM} \mathrm{MgCl}$, 5 mM EGTA, $1 \mathrm{mM}$ dithiothreitol, $100 \mu \mathrm{M}$ NaVO4, and 1\% NP40, in addition to protease inhibitors. Whole cell lysates $(20 \mu \mathrm{g})$ were immunoblotted and signals were detected with enhanced chemilumisescence (Thermo Fisher Scientific) and quantified in a Lumilmager (Roche Molecular Biochemicals).

\section{Identification of OSBPL5 and OSBPL8 homologs/orthologs in C. Elegans}

FASTA sequences for human OSBPL5 and OSBPL8 were obtained from the NCBI protein database. Subsequently, using the blast tool in WormBase (https:/ / wormbase.org/tools/blast_blat), homologs/ orthologs of OSBPL5 and OSBPL8 were identified. Hits with a percentage identity of 30 and above were considered as candidate genes.

\section{C. elegans vulva quantification assay}

RNAi-mediated knockdown of osbpl5 and osbpl8 was induced by feeding let-60(n1046) worms with Escherichia coli HT115 generating
dsRNA to target genes from their L1 stage to adult stage. A DIC (Differential Interface Contrast)/Nomarski microscope was used to score the MUV phenotype.

\section{Generation of CRISPR/Cas9 cell line}

CaCO-2 cells were transduced with OSBPL8 SgRNA (3'-TGCAAATCTTTGGTTGGCGT-5') plus Cas9 expression followed by puromycin selection $(4 \mu \mathrm{g} / \mathrm{ml})$ after $24 \mathrm{~h}$. Single colonies were generated from the pool of polyclonal $\mathrm{KO}$ cells.

\section{Lentiviral transduction}

For lentivirus production, TLA293T cells were transfected with ViraPower lentiviral packaging mix (K4975-00) using Lipofectamine (18324-012) and PLUS Reagent (10964-021). All reagents were purchased from Invitrogen. Lentiviral particles were collected 48 and $72 \mathrm{~h}$ after transfection, and then concentrated with Lenti-X concentrator (931232; Clontech). Titers were estimated with Lenti-X Go-Stix (\#631244; Clontech).

\section{shRNA knockdown and bicistronic transient infection}

OSBPL5 ShRNA was purchased from sigma-Aldrich (cat. no. SHCLNG-NM_020896, shRNAa: NM_020896.2-1316s1c1: 3'-CCGGGAACAAGCTCTCCGACTACTACTCGAGTAGTAGTCGGAGAGCTTGTTCTTTTTTG-5', shRNAb: NM_020896.2-2980s1c1: 3'-CCGGGCCTTAATGCTAAAGCCAAATCTCGAGATTTGGCTTTAGCATTAAGGCTTTTTTG-5', and shRNAc: NM_020896.2-2732s1c1: 3'- CCGGGTTCATTAACCACATCCTCAACTCGAGTTGAGGATGTGGTTAATGAACTITTTG-5'). shRNA constructs for OSBPL5 were cloned into pLenti6.3-V5-TOPO vector (K5315-20; Invitrogen). OSBPL8 shRNA pre-packaged into transduction particles was purchased from Dharmacon (cat. no. V3SH7602-226843976: 3'TGACAAGCCTATAAACACC-5'). The empty pLKO.1-TRC cloning vector was a gift from David Root (plasmid \#10878; Addgene). GFP-KG12V/ mCherry-CAAX and GFP-LactC2/mCherry-CAAX bicistronic plasmids were generated in-house and packaged into lentiviral particles. Pancreatic cancer cells were seeded at $4 \times 10^{5}$ cells per well in sixwell plates, infected with lentiviral particles $24 \mathrm{~h}$ later at $70 \%$ confluency, and osbpl5 and osbpl8 knockdown stable cell lines of were selected for with puromycin $(4 \mu \mathrm{g} / \mathrm{ml})$.

\section{Confocal microscopy}

Cells were seeded onto coverslips and allowed to grow for $48 \mathrm{~h}$ before fixation with $4 \%$ PFA and quenching with $50 \mathrm{mM} \mathrm{NH} \mathrm{NLl}_{4} \mathrm{C}$. Coverslips were then mounted in Mowiol and visualized by confocal microscopy (Nikon A1R) using a 60X objective.

\section{EM and spatial mapping}

Basal PM sheets of CaCO-2 and MDCK cells were prepared, fixed with $4 \%$ PFA and $0.1 \%$ glutaraldehyde, and labeled with affinity-purified anti-GFP antisera conjugated to $4.5-\mathrm{nm}$ gold as described previously (Prior et al, 2003). Digital images of immunolabeled membrane sheets were taken with a transition electron microscope at $100,000 \times$ magnification and intact $1-\mu \mathrm{m}^{2}$ areas were identified with ImageJ. $(x, y)$ coordinates of the gold particles were determined 
as described in Prior et al (2003). Univariate K function (Ripley, 1977) was calculated and standardized on a $99 \%$ confidence interval (Diggle et al, 2000; Hancock and Prior, 2005; Prior et al, 2003), whereby an $L(r)-r$ value greater than the confidence interval is indicative of significant clustering. The extent of clustering is represented by the (Lmax) value, the maximum value of the $\mathrm{K}$ function. Bootstrap tests were used to analyze differences between replicated point patterns as described previously (Diggle et al, 2000), and statistical significance was determined by evaluation against 1,000 bootstrap samples.

\section{Proliferation assay}

For shRNA knockdown studies, BxPC-3, PANC-1, MiaPaCa-2, and $\mathrm{MOH}$ parental and knockdown cells were seeded at a density of $2 \times 10^{5}$ cells/well in six-well plates and counted every day for $5 \mathrm{~d}$ using the countess automated cell counter (Invitrogen). For drug treatment studies as validated by Raubo et al (2015), HPNE ( $5 \times$ $\left.10^{3}\right), \mathrm{BxPC}-3\left(4 \times 10^{3}\right), \mathrm{PANC}-1\left(4 \times 10^{3}\right), \mathrm{MiaPaCa}-2\left(2 \times 10^{3}\right)$, and $\mathrm{MOH}$ $\left(1.5 \times 10^{3}\right)$ cells were seeded in 96 -well plates. After $24 \mathrm{~h}$, fresh growth medium supplemented with $1 \%$ DMSO or differing drug concentrations were added, and the cells were allowed to grow for another $72 \mathrm{~h}$. Cell numbers were determined by CyQuant Proliferation Assay (Thermo Fisher Scientific) according to the manufacturer's protocol.

\section{Anchorage-independent growth assay}

BxPC-3 $\left(10 \times 10^{3}\right)$, PANC-1 $\left(5 \times 10^{3}\right)$, MiaPaCa-2 $\left(5 \times 10^{3}\right)$, and $\mathrm{MOH}$ $\left(5 \times 10^{3}\right)$ parental and knockdown cells were seeded in soft agar in six-well plates, with a base layer of $1 \%$ agar-media mixture, and a top layer of $0.6 \%$ agar-cell suspension mix as performed in (Borowicz et al, 2014). After 2-3 wk, colonies were stained with $0.01 \%$ crystal violet and imaged. Colony numbers were quantified by ImageJ.

\section{Bioinformatic analysis using UCSC Xena browser}

ORP5 and ORP8 mRNA expression and KRAS mutational status in patients and their overall survival were analyzed and visualized using data in GDC TCGA-PAAD, TCGA-LUNG, and GDC-PANCAN, by Xena browser (https://xenabrowser.net/) (Goldman et al, 2019 Preprint).

\section{Statistical analysis}

Results are presented as the mean \pm SEM. Prism version 5.0 (GraphPad Software) was used for two-tailed $t$ test. Levels of significance are labeled as: ${ }^{\star} P<0.05$; ${ }^{\star \star} P<0.01$; ${ }^{* \star} P<0.001$; ${ }^{* * *} P<$ 0.0001 .

\section{Supplementary Information}

Supplementary Information is available at https://doi.org/10.26508/lsa. 201900431.

\section{Acknowledgements}

This Research was supported by Cancer Prevention and Research Institute of Texas (CPRIT) grant RP170233 to JF Hancock.

\section{Author Contributions}

WE Kattan: conceptualization, formal analysis, validation, investigation, visualization, methodology, and writing-original draft, review, and editing.

W Chen: validation and investigation.

$X$ Ma: investigation.

TH Lan: investigation.

D van der Hoeven: investigation.

$\mathrm{R}$ van der Hoeven: investigation.

JF Hancock: conceptualization, supervision, funding acquisition, project administration, and writing-review and editing.

\section{Conflict of Interest Statement}

The authors declare that they have no conflict of interest.

\section{References}

Balla A, Tuymetova G, Tsiomenko A, Várnai P, Balla T (2005) A plasma membrane pool of phosphatidylinositol 4-phosphate is generated by phosphatidylinositol 4-kinase type-III alpha: Studies with the PH domains of the oxysterol binding protein and FAPP1. Mol Biol Cell 16: 1282-1295. doi:10.1091/mbc.e04-07-0578

Borowicz S, Van Scoyk M, Avasarala S, Karuppusamy Rathinam MK, Tauler J, Bikkavilli RK, Winn RA (2014) The soft agar colony formation assay. J Vis Exp 27: e51998. doi:10.3791/51998

Boura E, Nencka R (2015) Phosphatidylinositol 4-kinases: Function, structure, and inhibition. Exp Cell Res 337: 136-145. doi:10.1016/j.yexcr.2015.03.028

Cho K-J (2016) Inhibition of acid sphingomyelinase depletes cellular phosphatidylserine and mislocalizes K-Ras from the plasma membrane. Mol Cell Biol 36: 363-374. doi:10.1128/MCB.00719-15

Chung J, Torta F, Masai K, Lucast L, Czapla H, Tanner LB, Narayanaswamy P, Wenk MR, Nakatsu F, De Camilli P (2015) INTRACELLULAR TRANSPORT. $\mathrm{PI} 4 \mathrm{P} /$ phosphatidylserine countertransport at ORP5- and ORP8mediated ER-plasma membrane contacts. Science 349: 428-432. doi:10.1126/science.aab1370

Clayton EL, Minogue S, Waugh MG (2013) Mammalian phosphatidylinositol 4kinases as modulators of membrane trafficking and lipid signaling networks. Prog Lipid Res 52: 294-304. doi:10.1016/j.plipres.2013.04.002

Cox AD, Fesik SW, Kimmelman AC, Luo J, Der CJ (2014) Drugging the undruggable RAS: Mission possible? Nat Rev Drug Discov 13: 828-851. doi:10.1038/nrd4389

Diggle PJ, Jorge Mateu J, Clough HE (2000) A comparison between parametric and non-parametric approaches to the analysis of replicated spatial point patterns. Adv Appl Probab 32: 331-343. doi:10.1017/ s0001867800009952

Filseck JM, Čopič A, Delfosse V, Vanni S, Jackson CL, Bourguet W, Drin G (2015) Phosphatidylserine transport by OPR/Osh proteins is driven by PI4P. Science 349: 432-436. doi:10.1126/science.aab1346.

Galmes R, Houcine A, van Vliet AR, Agostinis P, Jackson CL, Giordano F (2016) ORP5/ORP8 localize to endoplasmic reticulum-mitochondria 
contacts and are involved in mitochondrial function. EMBO Rep 17: 800-810. doi:10.15252/embr.201541108

Ghai R, Du X, Wang H, Dong J, Ferguson C, Brown AJ, Parton RG, Wu JW, Yang H (2017) ORP5 and ORP8 bind phosphatidylinositol-4, 5-biphosphate (PtdIns(4,5)P 2) and regulate its level at the plasma membrane. Nat Commun 8: 757. doi:10.1038/s41467-017-00861-5

Goldman M, Craft B, Hastie M, Repečka K, Kamath A, McDade F, Rogers D, Brooks AN, Zhu J, Haussler D (2019) The UCSC Xena platform for public and private cancer genomics data visualization and interpretation. bioRxiv doi:10.1101/326470Preprint posted May 30, 2018.

Hammond GRV, Fischer MJ, Anderson KE, Holdich J, Koteci A, Balla T, Irvine RF (2012) PI4P and PI(4,5)P2 are essential but independent lipid determinants of membrane identity. Science 337: 727-730. doi:10.1126/ science. 1222483

Hammond GRV, Machner MP, Balla T (2014) A novel probe for phosphatidylinositol 4-phosphate reveals multiple pools beyond the Golgi. J Cell Biol 205: 113-126. doi:10.1083/jcb.201312072

Hancock JF (2003) Ras proteins: Different signals from different locations. Nat Rev Mol Cell Biol 4: 373-384. doi:10.1038/nrm1105

Hancock JF, Parton RG (2005) Ras plasma membrane signalling platforms. Biochem J 389: 1-11. doi:10.1042/BJ20050231

Hancock JF, Paterson H, Marshall CJ (1990) A polybasic domain or palmitoylation is required in addition to the CAAX motif to localize p21ras to the plasma membrane. Cell 63: 133-139. doi:10.1016/0092-8674(90)90294-0

van der Hoeven D, Cho K, Zhou Y, Ma X, Chen W, Naji A, Montufar-Solis D, Zuo Y, Kovar SE, Levental KR, et al (2017) Sphingomyelin metabolism is a regulator of KRAS function. Mol Cell Biol 38: e00373-e00417. doi:10.1128/MCB.00373-17

Hancock JF, Prior IA (2005) Electron microscopic imaging of Ras signaling domains. Methods 37: 165-172. doi:10.1016/ j.ymeth.2005.05.018.Electron

Ishikawa S, Nagai Y, Masuda T, Koga Y, Nakamura T, Imamura Y, Takamori H, Hirota M, Funakosi A, Fukushima M, et al (2010) The role of oxysterol binding protein-related protein 5 in pancreatic cancer. Cancer Sci 101: 898-905. doi:10.1111/j.1349-7006.2009.01475.x

Kapoor A, Yao W, Ying H, Hua S, Liewen A, Wang Q, Zhong Y, Wu CJ, Sadanandam A, Hu B, et al (2014) Yap1 activation enables bypass of oncogenic KRAS addiction in pancreatic cancer. Cell 158: 185-197. doi:10.1016/j.cell.2014.06.003

Koga Y, Ishikawa S, Nakamura T, Masuda T, Nagai Y, Takamori H, Hirota M, Kanemitsu K, Baba Y, Baba H (2008) Oxysterol binding protein-related protein-5 is related to invasion and poor prognosis in pancreatic cancer. Cancer Sci 99: 2387-2394. doi:10.1111/j.1349-7006.2008.00987.x

Markowitz S, Willson JKV, Rago C, Cheong I, Lengauer C, Yun J, Vogelstein B, Schmidt K, Kinzler KW, Velculescu VE, et al (2009) Glucose deprivation contributes to the development of KRAS pathway mutations in tumor cells. Science 325: 1555-1559. doi:10.1126/science.1174229

Matsudaira T, Mukai K, Noguchi T, Hasegawa J, Hatta T, lemura SI, Natsume T, Miyamura N, Nishina H, Nakayama J, et al (2017) Endosomal phosphatidylserine is critical for the YAP signalling pathway in proliferating cells. Nat Commun 8: 1-11. doi:10.1038/s41467-017-01255-3

Miao B, Skidan I, Yang J, Lugovskoy A, Reibarkh M, Long K, Brazell T, Durugkar KA, Maki J, Ramana CV, et al (2010) Small molecule inhibition of phosphatidylinositol-3,4,5-triphosphate (PIP3) binding to pleckstrin homology domains. Proc Natl Acad Sci U S A 107: 20126-20131. doi:10.1073/pnas.1004522107

Murakoshi H, lino R, Kobayashi T, Fujiwara T, Ohshima C, Yoshimura A, Kusumi A (2004) Single-molecule imaging analysis of Ras activation in living cells. Proc Natl Acad Sci U S A 101: 7317-7322. doi:10.1073/ pnas.0401354101

Nakatsu F, Baskin JM, Chung J, Tanner LB, Shui G, Lee SY, Pirruccello M, Hao M, Ingolia NT, Wenk MR, et al (2012) Ptdins4P synthesis by PI4KIII $\alpha$ at the plasma membrane and its impact on plasma membrane identity. J Cell Biol 199: 1003-1016. doi:10.1083/jcb.201206095

Park Y, Park JM, Kim DH, Kwon J, Kim IA (2017) Inhibition of PI4K IIIa radiosensitizes in human tumor xenograft and immune-competent syngeneic murine tumor model. Oncotarget 8: 110392-110405. doi:10.18632/oncotarget.22778

Plowman SJ, Muncke C, Parton RG, Hancock JF (2005) H-ras, K-ras, and inner plasma membrane raft proteins operate in nanoclusters with differential dependence on the actin cytoskeleton. Proc Natl Acad Sci U S A 102: 15500-15505. doi:10.1073/pnas.0504114102

Prior IA, Lewis PD, Mattos C (2012) A comprehensive survey of ras mutations in cancer. Cancer Res 72: 2457-2467. doi:10.1158/0008-5472.CAN-11-2612

Prior IA, Muncke C, Parton RG, Hancock JF (2003) Direct visualization of ras proteins in spatially distinct cell surface microdomains. J Cell Biol 160: 165-170. doi:10.1083/jcb.200209091

Raubo P, Andrews DM, McKelvie JC, Robb GR, Smith JM, Swarbrick ME, Waring MJ (2015) Discovery of potent, selective small molecule inhibitors of $\alpha$-subtype of type III phosphatidylinositol-4-kinase (PI4KIII $)$. Bioorg Med Chem Lett 25: 3189-3193. doi:10.1016/ j.bmcl.2015.05.093

Ripley BD (1977) Modelling spatial patterns. J R Stat Soc Ser B 39: 172-212. doi:10.1111/j.2517-6161.1977.tb01615.x

Rowinsky EK (2006) Lately, it occurs to me what a long, strange trip it's been for the farnesyltransferase inhibitors. J Clin Oncol 24: 2981-2984. doi:10.1200//CO.2006.05.9808

Sebti SM, Der CJ (2003) Searching for the elusive targets of farnesyltransferase inhibitors. Nat Rev Cancer 3: 945-951. doi:10.1038/ $\operatorname{nrc1234}$

Singh A, Greninger P, Rhodes D, Koopman L, Violette S, Bardeesy N, Settleman J (2009) A gene expression signature associated with "K-Ras addiction" reveals regulators of EMT and tumor cell survival. Cancer Cell 15: 489-500. doi:10.1016/j.ccr.2009.03.022

Smith MM, Levitan DJ (2004) The Caenorhabditis elegans homolog of the putative prostate cancer susceptibility gene ELAC2, hoe-1, plays a role in germline proliferation. Dev Biol 266: 151-160. doi:10.1016/ j.ydbio.2003.10.016

Sohn M, Ivanova P, Brown HA, Toth DJ, Varnai P, Kim YJ, Balla T (2016) Lenz Majewski mutations in PTDSS1 affect phosphatidylinositol 4phosphate metabolism at ER-PM and ER-Golgi junctions. Proc Natl Acad Sci U S A 113: 4314-4319. doi:10.1073/pnas.1525719113

Sohn M, Zewe JP, Wills RC, Hammond GRV, Humpolickova J, Vrzal L, Chalupska D, Veverka V, Fairn GD, Boura E, et al (2018) PI(4,5)P2 controls plasma membrane PI4P and PS levels via ORP5/8 recruitment to ER-PM contact sites. J Cell Biol 217: 1797-1813. doi:10.1083/jcb.201710095

Tian T, Harding A, Inder K, Plowman S, Parton RG, Hancock JF (2007) Plasma membrane nanoswitches generate high-fidelity Ras signal transduction. Nat Cell Biol 9: 905-914. doi:10.1038/ncb1615

Vance JE, Steenbergen R (2005) Metabolism and functions of phosphatidylserine. Prog Lipid Res 44: 207-234. doi:10.1016/ j.plipres.2005.05.001

Vartanian S, Bentley C, Brauer MJ, Li L, Shirasawa S, Sasazuki T, Kim JS, Haverty P, Stawiski E, Modrusan Z, et al (2013) Identification of mutant K-Rasdependent phenotypes using a panel of isogenic cell lines. J Biol Chem 288: 2403-2413. doi:10.1074/jbc.M112.394130

Venditti R, Rega LR, Masone MC, Santoro M, Polishchuk E, Sarnataro D, Paladino S, D'Auria S, Varriale A, Olkkonen VM, et al (2019) Molecular determinants of ER-Golgi contacts identified through a new FRET-FLIM system. J Cell Biol 218: 1055-1065. doi:10.1083/jcb.201812020

Waring MJ, Andrews DM, Faulder PF, Flemington V, McKelvie JC, Maman S, Preston M, Raubo P, Robb GR, Roberts K, et al (2014) Potent, selective small molecule inhibitors of type III phosphatidylinositol-4-kinase $\alpha$ but not $\beta$-inhibit the phosphatidylinositol signaling cascade and 
cancer cell proliferation. Chem Commun (Camb) 50: 5388-5390. doi:10.1039/C3CC48391F

Waters AM, Der CJ (2018) KRAS: The critical driver and therapeutic target for pancreatic cancer. Cold Spring Harb Perspect Med 8: 1-18. doi:10.1101/ cshperspect.a031435

Weinberg F, Schulze E, Fatouros C, Schmidt E, Baumeister R, Brummer T (2014) Expression pattern and first functional characterization of riok-1 in Caenorhabditis elegans. Gene Expr Patterns 15: 124-134. doi:10.1016/ j.gep.2014.05.005

Yuan TL, Amzallag A, Bagni R, Yi M, Afghani S, Burgan W, Fer N, Strathern LA, Powell K, Smith B, et al (2018) Differential effector engagement by oncogenic KRAS. Cell Rep 22: 1889-1902. doi:10.1016/ j.celrep.2018.01.051

Zhou Y, Hancock JF (2015) Ras nanoclusters: Versatile lipid-based signaling platforms. Biochim Biophys Acta 1853: 841-849. doi:10.1016/ j.bbamcr.2014.09.008
Zhou Y, Liang H, Rodkey T, Ariotti N, Parton RG, Hancock JF (2014) Signal integration by lipid-mediated spatial cross talk between Ras nanoclusters. Mol Cell Biol 34: 862-876. doi:10.1128/MCB.01227-13

Zhou Y, Prakash P, Gorfe AA, Hancock JF (2018) Ras and the plasma membrane: A complicated relationship. Cold Spring Harb Perspect Med 8: a031831. doi:10.1101/cshperspect.a031831

Zhou Y, Prakash P, Liang H, Cho KJ, Gorfe AA, Hancock JF (2017) Lipid-sorting specificity encoded in K-Ras membrane anchor regulates signal output. Cell 168: 239-251.e16. doi:10.1016/j.cell.2016.11.059

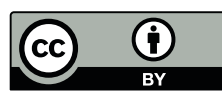

License: This article is available under a Creative Commons License (Attribution 4.0 International, as described at https://creativecommons.org/ licenses/by/4.0/). 